\title{
Diabetes management during Ramadan amid Covid-19 pandemic
}

\author{
Ali Tootee ${ }^{1} \cdot$ Ensieh Nasli Esfahani ${ }^{1} \cdot$ Bagher Larijani $^{2,3}$
}

Received: 21 May 2020 / Accepted: 23 June 2020 / Published online: 25 June 2020

(C) Springer Nature Switzerland AG 2020

\begin{abstract}
Management of fasting patients with diabetes during Ramadan has always posed a great challenge on clinicians. This year, Ramadan has coincided with the Covid-19 pandemic which per se can complicate diabetes care. Although patients with diabetes should be generally discouraged from fasting, those who insist to fact during the current Covid-19 pandemic should undergo strict risk assessment and receive thorough education. In patients with type 2 diabetes, administered antidiabetics and their dose should be modified to minimize the risk of hypoglycemia and dehydration. In type 1 diabetic patients, insulin dose and it timing should be precisely calculated based on regular and rigorous blood glucose monitoring. Nonetheless, it would be prudent to generally discourage patients with diabetes form fasting this Ramadan to avoid the risk of life-threatening complications such severe dehydration and ensuing kidney damage.
\end{abstract}

Keywords Diabetes $\cdot$ Covid-19 $\cdot$ Ramadan $\cdot$ Fasting

\section{Introduction}

Ramadan fasting is one of the main pillars of Islam, and Muslims without any adverse health condition need to fast from dawn to sunset during the whole month. Although fasting is compulsory for all Muslims, those who are pregnant, lactating, travelling, or have any acute or chronic health conditions such as diabetes are exempted from the ritual. Nonetheless, not all patients with diabetes are exempted, and those with satisfactory diabetes control may be allowed to fast. On the contrary, patients with poorly-controlled diabetes are at a very high risk for development of several life-threatening complications such as hypoglycemia, hyperglycemia, and ketoacidosis should refrain from fasting. [1]

This year, Ramadan has coincided with the Covid-19 Pandemic. It is documented that patients with diabetes are more

Bagher Larijani

emrc@tums.ac.ir

1 Diabetes Research Center, Endocrinology and Metabolism Clinical Sciences Institute, Tehran University of Medical Sciences, Tehran, Iran

2 Endocrinology and Metabolism Research Center, Endocrinology and Metabolism Clinical Sciences Institute, Tehran University of Medical Sciences, Tehran, Iran

3 Endocrinology and Metabolism Research Institute, Next to Shariati Hospital, Jalale Ale Ahmad Freeway, Amir Abad, Tehran, Iran susceptible to development of severe complications of Covid-19 such as Adult Respiratory Distress Syndrome and multi-organ failure. [2] In the elderly with type 2 diabetes, in particular, COVID-19 can lead to severe hyperglycemia with lifethreatening consequences. [3] Moreover, results of several studies suggest that people with diabetes are at an increased risk for contracting the infection. [4] Therefore, during the current Ramadan, clinical decision making in regards with the management of diabetic patients who wish to fast could be really challenging for clinicians. It should be stressed here, however, that patients with active covid-19 infection should not fast and should refrain from fasting for several weeks after complete recovery. [5]

\section{Risk assessment and patient education}

For patients with diabetes who intend to fast, risk assessment is of crucial importance. In this regard, general state of the health of the patients should be taken into consideration, and factors such as glycemic control, blood pressure, and lipids profile should be evaluated through appropriate blood studies and other investigations. [1] Subsequently, medical recommendations should be formulated in a tailored-made manner for each individual patient, and they should be informed of the potential risks they are accepting. They should be particularly be warned that any bacterial or viral infection including Covid-19 may severely affect blood sugar control. [6] The 
risk of dehydration and its grave consequences should also be especially emphasized. [2] During this assessment, diet and medications of patients should be carefully reviewed and considered in the ultimate decision. In this stage, people with diabetes experiencing any symptom of Covid-19, (such as high fever, dry cough, anosmia, loss of appetite,...) should be considered as high-risk and discouraged from fasting. It should also be noted that those with positive Covid-19 test results or patients who have recently recovered from the disease may be at a greater risk for development of diabetes complications for several weeks. [2]

Provision of necessary information for both the patients and their families should be the next step. This education should include advise and recommendation on basic diabetes care such as signs and symptoms of hyper-and hypoglycemia, blood glucose monitoring, meal planning, physical activity, medication administration, and management of possible acute complications. Frequent blood glucose monitoring is imperative for patients with diabetes who choose to fast, and these patients should be properly educated in this regard. Clinicians should particularly mention the symptom of Covid-19 to their patients, and patients need to be advised to immediately stop fasting and ensure adequate hydration should the symptoms develop. Particularly, proper hydration should be strictly recommended as Covid-19 is per se a great cause of dehydration in patients with diabetes. [6] Adequate nutrition and hydration during non-fasting hours should also be emphasized, and prompt treatment of hypoglycemia with glucose-rich food (or supplements) need to be considered. [1] In patients at higher risks, glucagon injection may also be taught to partners and family members. [1]

\section{Management of patients with type 2 diabetes}

Patients with type 2 diabetes who are satisfactorily managed through only dietary interventions and exercise are generally at lower risk for development of diabetes complications which may be exacerbated by Covid-19. Elderly patients with type 2 diabetes who are inflicted with Covid-19 are nevertheless at an increased risk. [3] Moreover, in this group of patients, there exists an accentuated risk for occurrence of severe postprandial hyperglycemia after the predawn and sunset meals if they consume huge calories. [1] Besides, considering the fact that diabetic patients in advanced ages often simultaneously suffer from hypertension and dyslipidemia, fasting-induced dehydration which may be exacerbated by Covid- 19 symptoms can dramatically increase the risk of thrombotic events. [2] Generally, those on diabetes medications should be managed on an individual basis. Anti-diabetic medications can be generally divided into two categories of high-risk and low-risk drugs depending on the risk of hypoglycemia they pose. (Table 1).

Patients who are adequately managed by metformin are generally at a lower risk of complications, and their possibility of development of hypoglycemia is considerably low. However, in Ramadan, the timing of the metformin doses need to be adjusted. It is generally recommended that two thirds of the total daily dose should be administered immediately before Iftar, and the other third needs to be given before Sahar. [1] Nonetheless, should Covid-19 symptoms appear, patients need to immediately stop taking metformin and ensure adequate hydration. [2] In critical situations, renal function should also be carefully monitored as there exist a very high risk of damage to the kidneys if severe dehydration ensues.

Patients on insulin sensitizers (rosiglitazone and pioglitazone) are generally at low risk for development of hypoglycemia, and they may continue their medication while fasting. [1] Patients on glucagon-like peptide-1 receptor agonists (such as albiglutide, dulaglutide,...), nonetheless, need close monitoring and adequate hydration during non-fasting hours as dehydration may lead to serious consequences. [2] Dipeptidyl peptidase-4 inhibitors (such as alogliptin, linagliptin, saxagliptin, ...) are generally well tolerated and do not need any alteration during Ramadan. [2] Conversely, sulfonylureas are not recommendable during Ramadan because of the risk for development of hypoglycemia, and, if needs be, they should be used with extreme caution. [5] Naturally, during the current pandemic, it would be tenable that they should be totally avoided due to the high risk of development of hypoglycemia. [7] The use of sodiumglucose-co-transporter 2 inhibitors (such as canagliflozin, dapagliflozin, and empagliflozin) during Ramadan is controversial, nonetheless they should be avoided due to high risk of development of dehydration, diabetic ketoacidosis, and consequent kidney damages in the context of Covid-19. [1, 8, 9]

As patients with diabetes (particularly the elderly with type 2 diabetes) simultaneously consume anti-hypertensive and lipid lowering medications, dose and timing of these drug may also need to be modified in the context of Covid-19 pandemic. Angiotensin-converting enzyme inhibitors (ACEIs) and angiotensin receptor blockers (ARBs) are two commonly-used class of medications with widespread use for management of hypertension and related consequences. Some studies have suggested that there may be a link between consumption of ACEIs and ARBs and increased risk for contracting Covid-19. [10] It is assumed that the underlying mechanism of this relationship is the documented association between angiotensin-converting enzyme 2 (ACE2) and SARS-CoV-2. ACE2 is demonstrated to be a co-receptor for the entry of SARS-CoV-2, and some suggest that it may have role in the pathogenesis of COVID-19. In this regard, currently, there exists no conclusive evidence in favor or against this hypothesis, and most clinical guideline have not hitherto issued any statement as to the necessity of discontinuation of this class of anti-hypertensive medications during the Covid-19 Pandemic. [10] As regards ReninAngiotensin-Aldosterone System Inhibitors (RAAIs), there are concerns that they might enhance contraction or increase 
Table 1 Recommended changes to treatment regimen in patients with diabetes who fast during Ramadan in the context of covid19 pandemic

Diabetes medications

General considerations in Ramadan in the context of COVID-19 pandemic

Metformin

Sodium-glucose-co-transporter 2 inhibitors (canagliflozin, dapagliflozin, and empagliflozin, ...)

Glucagon-like peptide-1 receptor agonists (albiglutide, dulaglutide, exenatide-extended release, liraglutide,

lixisenatide, and semaglutide,...)

Dipeptidyl peptidase-4 inhibitors (alogliptin, linagliptin, saxagliptin, and sitagliptin...)

Sulfonylureas

Insulin
- Patients on metformin may safely fast because of the low risk of severe hypoglycemia

- In the context of Covid-19, dehydration can lead to lactic acidosis

- In fasting patients on metformin, the risk of chronic kidney disease or acute kidney injury is high in the context of Covid-19

- Patients should immediately stop fasting and discontinue metformin on appearance of any symptom attributable to Covid-1

- Due to the low risk of hypoglycemia, they are generally considered safe in Ramadan

- There is increased risk of dehydration and diabetic ketoacidosis in Covid-19 complications

- Patients should immediately stop taking them if any associated symptom develops

- There is an increased risk for acute kidney injury in Covid-19 infection

- They may be safely used during Ramadan

- Dehydration caused by Covid-19 may lead to serious complications

- They should be stopped and adequate hydration needs to be immediately followed should any related symptom develop

- The risk for development of hypoglycemia and other complications is relatively low in fasting

- They can be safely used during Ramadan

- These drugs are generally considered safe in Covid-19 infection

- They are unsuitable for use during fasting because of the high risk of hypoglycemia.

- Insulin therapy should be continued during fasting and Covid-19 infection

- Dose of insulin should be guided by regular self-monitoring of blood glucose levels every $2-4 \mathrm{~h}$

- If severe complications of Covid-19 unfold, higher doses of insulin may be needed to achieve normoglycemia severity of Covid-19. Nonetheless, most guidelines contend that RAAIs should be continued in patients in stable conditions during Covid-19 pandemic. [11] Patients who consume other classes of medication such as diuretics and NSAIDS should consult with their clinicians as to continuation or cessation of these drugs because of the serious risk of dehydration and kidney damage they may pose. [6]

\section{Management of patients with type 1 diabetes}

For patients with type 1 diabetes who plan to fast, intensive glycemia management is of paramount importance during the current Covid-19 Pandemic. This can be achieved through several daily insulin injections (three or more) guided by regular capillary blood glucose measurement, which should be performed at least four times a day: at Sahar time, $2 \mathrm{~h}$ into the fast, and before the Iftar meal. [12] This Ramadan, patients may need higher daily doses of insulin, and may even need to be administered intravenous insulin infusion should severe complications such as respiratory failure and sepsis develop. [13] Most patients, nonetheless, are successfully managed by means of two doses of NPH before Sahar and Iftar meals, along with a short-acting insulin preparation which should be administered according to daily blood glucose levels. [12] 


\section{Conclusion}

To sum up, it may be concluded that as fasting patients with Covid-19 are at a dual risk for development of diabetes complications, they should be generally advised against fasting this Ramadan. In fasting individuals, the risk of development of dehydration and severe hypoglycemia is great and the consequences can be life-threatening in the context of Covid-19 infection. On the other hand, the need for increased doses of insulin and loss of appetite in these patients as a result of Covid-19 may per se lead to poor glycemic control and diabetic ketoacidosis particularly in the elderly patients. Therefore, from a pragmatical viewpoint, although patients' determination for fasting should be respected and taken into consideration, avoiding the risk of severe complications should be in priority for practicing clinicians during the current pandemic. Nonetheless if patients still persevere with their divine obligation for any spiritual, social, or ideological reason, they should receive proper education and follow comprehensive instructions regarding exercise, nutrition, regular glucose monitoring, and use of antidiabetic medications. Therefore, it can be contended that approach to management of diabetic patients who intend to fast during the current Covid-19 pandemic should be uniquely individualized, and these patients should be closely monitored for potential lifethreatening complications.

\section{References}

1. Al-Arouj $\mathrm{M}$, et al. Recommendations for management of diabetes during Ramadan: update 2010. Diabetes Care. 2010;33(8):1895902.

2. Bornstein, S.R., et al. Practical recommendations for the management of diabetes in patients with COVID-19. The Lancet Diabetes \& Endocrinology. 2020.
3. Xue, T., et al. Blood glucose levels in elderly subjects with type 2 diabetes during COVID-19 outbreak: a retrospective study in a single center. Available at SSRN 3566198, 2020.

4. Hassanein M, al Awadi FF, el Hadidy KES, Ali SS, Echtay A, Djaballah K, et al. The characteristics and pattern of care for the type 2 diabetes mellitus population in the MENA region during Ramadan: an international prospective study (DAR-MENA T2DM). Diabetes Res Clin Pract. 2019;151:275-84.

5. Hanif S, Ali SN, Hassanein M, Khunti K, Hanif W. Managing people with diabetes fasting for Ramadan during the COVID-19 pandemic: a South Asian Health Foundation update. Diabet Med. 2020;37:1094-102.

6. Sick day rules: how to manage Type 2 diabetes if you become unwell with coronavirus and what to do with your medication. 2020; Available from: https://www.england.nhs.uk/london/wpcontent/uploads/sites/8/2020/04/3.-Covid-19-Type-2-Sick-DayRules-Crib-Sheet-06042020.pdf.

7. DeWitt CR, Heard K, Waksman JC. Insulin \& C-peptide levels in sulfonylurea-induced hypoglycemia: a systematic review. J Med Toxicol. 2007;3(3):107-18.

8. Bashier A, Khalifa AA, Abdelgadir EI, al Saeed MA, al Qaysi AA, Ali Bayati MB, et al. Safety of sodium-glucose cotransporter 2 inhibitors (SGLT2-I) during the month of Ramadan in muslim patients with type 2 diabetes. Oman Med J. 2018;33(2):104-10.

9. Hassanein M, Echtay A, Hassoun A, Alarouj M, Afandi B, Poladian R, et al. Tolerability of canagliflozin in patients with type 2 diabetes mellitus fasting during Ramadan: results of the Canagliflozin in Ramadan tolerance observational study (CRATOS). Int J Clin Pract. 2017;71(10):e12991.

10. Patel AB, Verma A. COVID-19 and angiotensin-converting enzyme inhibitors and angiotensin receptor blockers: what is the evidence? JAMA. 2020.

11. Vaduganathan M, Vardeny O, Michel T, McMurray JJV, Pfeffer MA, Solomon SD. Renin-angiotensin-aldosterone system inhibitors in patients with Covid-19. N Engl J Med. 2020;382(17):16539.

12. Al-Arouj M, et al. Recommendations for management of diabetes during Ramadan. Diabetes Care. 2005;28(9):2305-11.

13. Dellinger RP, et al. Surviving Sepsis campaign: international guidelines for management of severe sepsis and septic shock, 2012. Intensive Care Med. 2013;39(2):165-228.

Publisher's note Springer Nature remains neutral with regard to jurisdictional claims in published maps and institutional affiliations. 